\title{
Single-sided deafness and directional hearing: contribution of spectral cues and high-frequency hearing loss in the hearing ear
}

\author{
Martijn J. H. Agterberg ${ }^{1,2 *}$, Myrthe K. S. Hol ${ }^{2}$, Marc M. Van Wanrooij ${ }^{1,2}$, A. John Van Opstal ${ }^{1}$ and \\ Ad F. M. Snik ${ }^{1,2}$ \\ ' Department of Biophysics, Donders Institute for Brain, Cognition and Behaviour, Radboud University Nijmegen, Nijmegen, Netherlands \\ ${ }^{2}$ Department of Otorhinolaryngology, Donders Institute for Brain, Cognition and Behaviour, Radboud University Medical Center, Nijmegen, Netherlands
}

\author{
Edited by: \\ Guillaume Andeol, Institut de \\ Recherche Biomédicale des \\ Armées, France \\ Reviewed by: \\ Robert Baumgartner, Austrian \\ Academy of Sciences, Austria \\ Andrzej J. Zarowski, European \\ Institute for ORL-HNS, Belgium \\ *Correspondence: \\ Martijn J. H. Agterberg, Department \\ of Biophysics, Donders Institute for \\ Brain, Cognition and Behaviour, \\ Radboud University Nijmegen, \\ Heyendaalseweg 135, \\ 6525 AJ Nijmegen, Netherlands \\ e-mail: m.agterberg@donders.ru.nl
}

Direction-specific interactions of sound waves with the head, torso, and pinna provide unique spectral-shape cues that are used for the localization of sounds in the vertical plane, whereas horizontal sound localization is based primarily on the processing of binaural acoustic differences in arrival time (interaural time differences, or ITDs) and sound level (interaural level differences, or ILDs). Because the binaural sound-localization cues are absent in listeners with total single-sided deafness (SSD), their ability to localize sound is heavily impaired. However, some studies have reported that SSD listeners are able, to some extent, to localize sound sources in azimuth, although the underlying mechanisms used for localization are unclear. To investigate whether SSD listeners rely on monaural pinna-induced spectral-shape cues of their hearing ear for directional hearing, we investigated localization performance for low-pass filtered ( $L P,<1.5 \mathrm{kHz}$ ), high-pass filtered $(\mathrm{HP},>3 \mathrm{kHz})$, and broadband $(\mathrm{BB}, 0.5-20 \mathrm{kHz})$ noises in the two-dimensional frontal hemifield. We tested whether localization performance of SSD listeners further deteriorated when the pinna cavities of their hearing ear were filled with a mold that disrupted their spectral-shape cues. To remove the potential use of perceived sound level as an invalid azimuth cue, we randomly varied stimulus presentation levels over a broad range (45-65 dB SPL). Several listeners with SSD could localize HP and BB sound sources in the horizontal plane, but inter-subject variability was considerable. Localization performance of these listeners strongly reduced after diminishing of their spectral pinna-cues. We further show that inter-subject variability of SSD can be explained to a large extent by the severity of high-frequency hearing loss in their hearing ear.

Keywords: azimuth, head-shadow effect, mold, single-sided deaf(ness), spectral pinna-cues

\section{INTRODUCTION}

Listeners with total single-sided deafness (SSD) lack the ability to localize sounds on the basis of interaural differences in time (ITD) and sound level (ILD). As a result, SSD listeners encounter significant problems with the processing of auditory information in daily life (Van Wieringen et al., 2011; Lieu, 2013), and demonstrate impaired sound-localization abilities (Humes et al., 1980; Colburn, 1982; Slattery and Middlebrooks, 1994; Bosman et al., 2003; Van Wanrooij and Van Opstal, 2004; Wazen et al., 2005). Similar effects have been reported for unilateral plugged control listeners (McPartland et al., 1997; Van Wanrooij and Van Opstal, 2007; Kumpik et al., 2010; Irving and Moore, 2011; Agterberg et al., 2012), and unilateral plugged experimental animals (Keating et al., 2013; Kral et al., 2013). Several studies, in which sound levels were fixed or varied over a small range,

Abbreviations: BB, broadband; HP, high-pass; HRTFs, head-related transfer functions; HSE, head-shadow effect; ILDs, interaural level differences; ITDs, interaural time differences; LP, low-pass; MAE, mean absolute error; SSD, single-sided deaf(ness). have demonstrated sound-localization abilities of SSD listeners (Batteau, 1967; Colburn, 1982; Häusler et al., 1983; Slattery and Middlebrooks, 1994; Wightman and Kistler, 1997). When stimuli are presented at a single sound level, SSD listeners could rely on the perceived sound level at the hearing ear because of the azimuth-dependent attenuation produced by the head-shadow effect (HSE). Van Wanrooij and Van Opstal (2004) demonstrated that the HSE indeed contributes to sound localization abilities of SSD listeners. Furthermore, the possibility that these listeners have learned to use monaural pinna-induced spectral-shape cues of their hearing ear for localization in azimuth, has been postulated (Batteau, 1967; Colburn, 1982; Häusler et al., 1983; Slattery and Middlebrooks, 1994; Wightman and Kistler, 1997; Van Wanrooij and Van Opstal, 2004; Shub et al., 2008; Kumpik et al., 2010; Rothpletz et al., 2012). The studies mentioned above did not take into account the hearing loss of the better ear, and included only subjects with a normal hearing ear (i.e., hearing thresholds $\leq 25 \mathrm{~dB}$ HL at frequencies between 0.25 and $8 \mathrm{kHz}$ ). Especially when stimuli contain high-frequencies information, 
monaural pinna-induced spectral-shape cues can be beneficial for localization (Best et al., 2005). Recently it has been reported that older listeners (63-80 years) with hearing loss above $5 \mathrm{kHz}$ demonstrated deteriorated sound localization in elevation as compared to normal hearing listeners (Otte et al., 2013). Highfrequency hearing loss did not affect sound localization abilities in azimuth. These results show that with advancing age and subsequent increasing high-frequency hearing loss, listeners lose the access to spectral-shape information for the localization of broadband (BB) stimuli in elevation. The loss of this ability might be of importance for listeners with SSD.

Animal studies have indicated that early onset of unilateral deafness results in a unilateral aural preference, reflected by local field potentials recorded from the cortical surface (Kral et al., 2013). Others, demonstrated that the ability to use spectral localization cues diminished as soon as normal hearing was experienced (Keating et al., 2013). As it is unclear whether a critical period for this auditory plasticity might also be present in humans, and it is postulated that the etiology of subjects with SSD may be unrelated to their localization abilities (Colburn, 1982), we investigated whether the onset of unilateral deafness (congenital vs. acquired) affects sound-localization performance in azimuth and elevation when tested at a later age.

Listeners with SSD demonstrate a large variability in their localization performance and it is not clear whether this variation is related to hearing loss, pinna-induced spectral shape cues, or to the onset of unilateral deafness. In the present study we investigated to what extent high-frequency hearing loss in the hearing ear of SSD listeners affects their use of spectralshape cues to localize sounds in azimuth. Furthermore, for SSD patients who are seeking hearing revalidation an improved number of treatment options have become available. It is important to identify the factors affecting sound localization abilities of SSD listeners. This information is helpful for clinicians in the search for the best possible treatment for listeners with monaural hearing.

\section{METHODS \\ LISTENERS WITH SSD AND CONTROL LISTENERS}

Nineteen listeners with complete SSD (16-67 years; mean $\pm S D$ : $40.7 \pm 16.7$ years) and 15 control listeners (22-61 years; mean $\pm S D: 30.9 \pm 12.4$ years) participated in the present study. Table 1 lists the characteristics of listeners with SSD and indicates which listeners experienced listening with a bone-conduction device. To assess hearing loss in the better ear, we performed puretone audiometry at $0.125,0.25,0.5,1,2,4$, and $8 \mathrm{kHz}$. Hearing thresholds were thus obtained using standard procedures and standard equipment (Interacoustics AC 40 clinical audiometer, Interacoustics A/S, Assens, Denmark).

\section{MOLD IN THE BETTER EAR}

The SSD listeners were tested in two hearing conditions that were presented in randomized order: (i) monaural hearing; (ii) monaural hearing with a custom-made mold, fabricated from rubber casting material (Otoform Otoplastik-K/c; Dreve, Unna, Germany), inserted in the pinna of the better-hearing ear without obstructing the ear canal.
All control listeners were tested under normal hearing conditions, and after altering their pinna-cues with custom-made molds in both pinna.

\section{STIMULI}

Listeners were asked to localize (i) low-pass (LP; $0.5-1.5 \mathrm{kHz}$ ); (ii) high-pass (HP; 3-20 kHz), and (iii) broadband (BB; $0.5-20 \mathrm{kHz})$ filtered Gaussian white noises. Spectral cues are minimal for LP noises (Middlebrooks and Green, 1991; Middlebrooks, 1992; Frens and Van Opstal, 1995; Blauert, 1997; Van Wanrooij and Van Opstal, 2004, 2007), and we therefore hypothesized that LP noises could not be localized in azimuth at all by SSD listeners.

$\mathrm{BB}$ and HP stimuli were chosen to maximize the use of potential spectral-shape cues provided by the pinna of the betterhearing ear. BB and HP stimuli had randomly-selected sound levels in the range 45-65 dB SPL. LP noises were interleaved with the BB and HP stimuli, and only presented at a level of $55 \mathrm{~dB}$ SPL. To minimize measurement time and because the attenuation of sound level by the head is not very effective for LP noises, we decided not to rove the levels of the LP stimuli.

All stimuli had 150-ms duration, 5-ms sine- and cosinesquared on- and offset ramps and a flat spectrum level within their pass bands. Sounds were digitally generated in Matlab (The MathWorks) at a sampling rate of $50 \mathrm{kHz}$, and were delivered through a $\mathrm{BB}$ loudspeaker, moved by a computer-controlled motorized system at a distance of $1.15 \mathrm{~m}$ from the listener's head. Stimulus coordinates for $\mathrm{BB}$ and $\mathrm{HP}$ stimuli ranged from $-85^{\circ}$ to $+85^{\circ}$ in azimuth and from $-30^{\circ}$ to $+30^{\circ}$ in elevation. LP stimuli were presented at $0^{\circ}$ in elevation.

Table 1 | Audiometric characteristics of the listeners with SSD.

\begin{tabular}{lcclcc}
\hline $\begin{array}{l}\text { SSD } \\
\text { patients }\end{array}$ & Age (y) & Side HL & $\begin{array}{l}\text { Congenital } \\
\text { acquired }\end{array}$ & Gender & $\begin{array}{c}\text { Threshold dB } \\
\text { HL 8 kHz }\end{array}$ \\
\hline P1 & 32 & $\mathrm{~L}$ & Congenital & $\mathrm{M}$ & 0 \\
P2 & 22 & $\mathrm{~L}$ & Congenital & $\mathrm{M}$ & 10 \\
P3 & 22 & $\mathrm{~L}$ & Congenital & $\mathrm{M}$ & 5 \\
P4 & 24 & $\mathrm{R}$ & Congenital & $\mathrm{V}$ & 10 \\
P5 & 51 & $\mathrm{~L}$ & Congenital & $\mathrm{M}$ & 65 \\
P6* & 46 & $\mathrm{~L}$ & Congenital & $\mathrm{V}$ & 10 \\
P7* & 27 & $\mathrm{R}$ & Congenital & $\mathrm{M}$ & 5 \\
P8 & 46 & $\mathrm{~L}$ & Congenital & $\mathrm{V}$ & 5 \\
P9* & 16 & $\mathrm{~L}$ & Congenital & $\mathrm{M}$ & 0 \\
P10* & 34 & $\mathrm{~L}$ & Congenital & $\mathrm{M}$ & 35 \\
P11 & 20 & $\mathrm{~L}$ & Congenital & $\mathrm{V}$ & 0 \\
P12 & 67 & $\mathrm{~L}$ & Acquired & $\mathrm{M}$ & 70 \\
P13 & 38 & $\mathrm{R}$ & Acquired & $\mathrm{V}$ & 20 \\
P14* & 53 & $\mathrm{R}$ & Acquired & $\mathrm{V}$ & 40 \\
P15* & 63 & $\mathrm{~L}$ & Acquired & $\mathrm{V}$ & 5 \\
P16 & 34 & $\mathrm{~L}$ & Acquired & $\mathrm{M}$ & 30 \\
P17 & 51 & $\mathrm{~L}$ & Acquired & $\mathrm{M}$ & 40 \\
P18 & 67 & $\mathrm{R}$ & Acquired & $\mathrm{M}$ & 55 \\
P19 & 60 & $\mathrm{~L}$ & Acquired & $\mathrm{M}$ & 60 \\
\hline *19 & & & & & \\
\hline
\end{tabular}

*Indicates listeners who experienced listening with a bone-conduction device. 


\section{SETUP}

For a detailed description of the setup see Bremen et al. (2010). Briefly, we ensured that listeners could only use acoustic information to localize sounds by testing directional hearing in a completely dark, sound-attenuated room. Horizontal and vertical head-movement components were recorded with the magnetic search-coil induction technique (Robinson, 1963; Hofman and Van Opstal, 1998). Listeners pointed with a head-fixed laser pointer, which projected onto a small $\left(1 \mathrm{~cm}^{2}\right)$ black plastic plate positioned in front $(40 \mathrm{~cm})$ of the listener's eyes. Listeners were asked to point the laser dot as fast and as accurately as possible in the perceived sound direction after stimulus exposure. Listeners were observed continuously by the experimenter with an infrared camera, but did not receive any feedback about their performance during the experiments.

\section{PARADIGM}

The experimental session started with a brief visual calibration experiment to establish the off-line mapping of the coil signals onto known target locations. After this, listeners performed a brief practice session containing 10 trials to become familiar with the head-movement response procedure.

During the sound-localization experiments the listener first fixated on an LED that was located at $0^{\circ}$ azimuth and $0^{\circ}$ elevation and then triggered the start of the trial by pressing a button. Between 150 and $300 \mathrm{~ms}$ the LED disappeared, and $200 \mathrm{~ms}$ later the sound stimulus was presented. After stimulus exposure the listener had to direct the head-fixed laser pointer as fast and accurately as possible, by making a rapid head movement toward the apparent sound direction.

\section{DATA ANALYSIS}

We analyzed the azimuth $(\alpha)$ responses separately for each stimulus condition (LP, HP, and BB noises) and for each listener. We determined the best linear fit (based on the mean-squared error criterion) of the stimulus-response relationship (pooled across presentation levels and elevation angles for $\mathrm{HP}$ and $\mathrm{BB}$ noises):

$$
\alpha_{R E S P}=b+g \cdot \alpha_{S T I M}
$$

where $\alpha_{R E S P}$ is the response azimuth (in degrees), $\alpha_{S T I M}$ is the stimulus azimuth (in degrees), $b$ is the response bias (in degrees), and $g$ the response gain (dimensionless). We also computed Pearson's correlation coefficient between fit and data, as well as the coefficient of determination $\left(r^{2}\right)$. To dissociate the potential contribution of the proximal sound level, $L$, from that of the actual stimulus location, we performed a partial correlation analysis:

$$
\widehat{\alpha}_{R E S P}=p \cdot \widehat{\alpha}_{S T I M}+q \cdot \hat{L}
$$

with $p$ as the dimensionless azimuth coefficient and $q$ as the dimensionless proximal sound-level coefficient; each determines to what extent sound-source azimuth or proximal sound level explains the observed responses. Variables $\alpha_{R E S P}, \alpha_{S T I M}$ and $L$ were transformed into their (dimensionless) z-scores $\hat{x}$ :

$$
\hat{x} \equiv \frac{x-\mu_{x}}{\sigma_{x}}
$$

with $x$ the variable to be $z$-transformed, $\mu_{x}$ its mean, and $\sigma_{x}$ its standard deviation (resulting in $\widehat{\alpha}_{R E S P}, \widehat{\alpha}_{S T I M}$, and $\hat{L}$ ). We determined proximal sound level $L$ by correcting the freefield presentation levels of the stimuli with the frequency- and azimuth-dependent attenuation produced by the HSE.

The HSE was derived for BB noises from the best fit of freefield HSE measurements of four listeners (Van Wanrooij and Van Opstal, 2004). For HP and BB noises the HSE can vary between -15 and $+15 \mathrm{~dB}$ over the entire azimuth range, for LP noises the HSE is less pronounced.

For the elevation $(\varepsilon)$ responses to $\mathrm{BB}$ and $\mathrm{HP}$ noises the best linear fits of the stimulus-response relationships were also determined.

$$
\varepsilon_{R E S P}=b+g \cdot \varepsilon_{S T I M}
$$

$\varepsilon_{\text {RESP }}$ and $\varepsilon_{S T I M}$ are the response elevation and stimulus elevation in degrees, $b$ is the response bias (in degrees) and $g$ the response gain (dimensionless).

\section{RESULTS}

\section{HIGH-FREOUENCY HEARING LOSS}

Normal hearing thresholds (defined as $20 \mathrm{~dB}$ HL or better) in the functioning ear were confirmed in all listeners with SSD $(n=19)$ for frequencies up to $4 \mathrm{kHz}$. At $8 \mathrm{kHz} 11 \mathrm{SSD}$ listeners demonstrated normal hearing. The other SSD listeners demonstrated thresholds $\geq 20 \mathrm{~dB}$ HL, with six listeners demonstrating thresholds $\geq 40 \mathrm{~dB}$ HL (see Table 1 ).

In the group of control listeners $(n=15)$, two older listeners (age 56 and 61 years) suffered from a symmetric hearing loss at $8 \mathrm{kHz}$ (thresholds $\geq 40 \mathrm{~dB} \mathrm{HL}$ ). All other control listeners demonstrated normal hearing thresholds between $500 \mathrm{~Hz}$ and $4 \mathrm{kHz}$, and thresholds of $40 \mathrm{~dB} \mathrm{HL}$, or better, for $8 \mathrm{kHz}$.

\section{EFFECT OF STIMULUS BANDWIDTH}

Figure 1A shows the stimulus-response relations in azimuth for a control listener (C1), and two listeners with SSD at their left side (P3 and P12), for BB, LP, and HP stimuli. For the BB and HP stimuli responses for the presentation levels $(45,55$, and $65 \mathrm{~dB}$ SPL) were pooled. The dashed lines represent the best-fit linear regression lines (Equation 1) on the azimuth response components. The control listener (right-hand column) could accurately localize stimuli for all conditions as is indicated by $r^{2}$ values and gains close to 1 . Note that perfect localization would mean that all individual responses would exactly be on the diagonal with slope +1.0 (with parameters: $r^{2}=1, g=1, b=0$ ). Listener P3 with SSD demonstrated good localization performance for BB and HP stimuli $\left(r^{2}>0.79 ; g>0.72 ; b\right.$ between $0^{\circ}$ and $\left.4^{\circ}\right)$. In contrast, listener P12 with SSD demonstrated poor soundlocalization abilities. This listener perceived the stimuli mainly at the hearing side, which resulted in a considerable leftward bias ( $b=80^{\circ}$ for $\mathrm{BB}$ stimuli), and small coefficients of determination $\left(r^{2}<0.10\right)$ for all stimuli and conditions. The hearing thresholds 


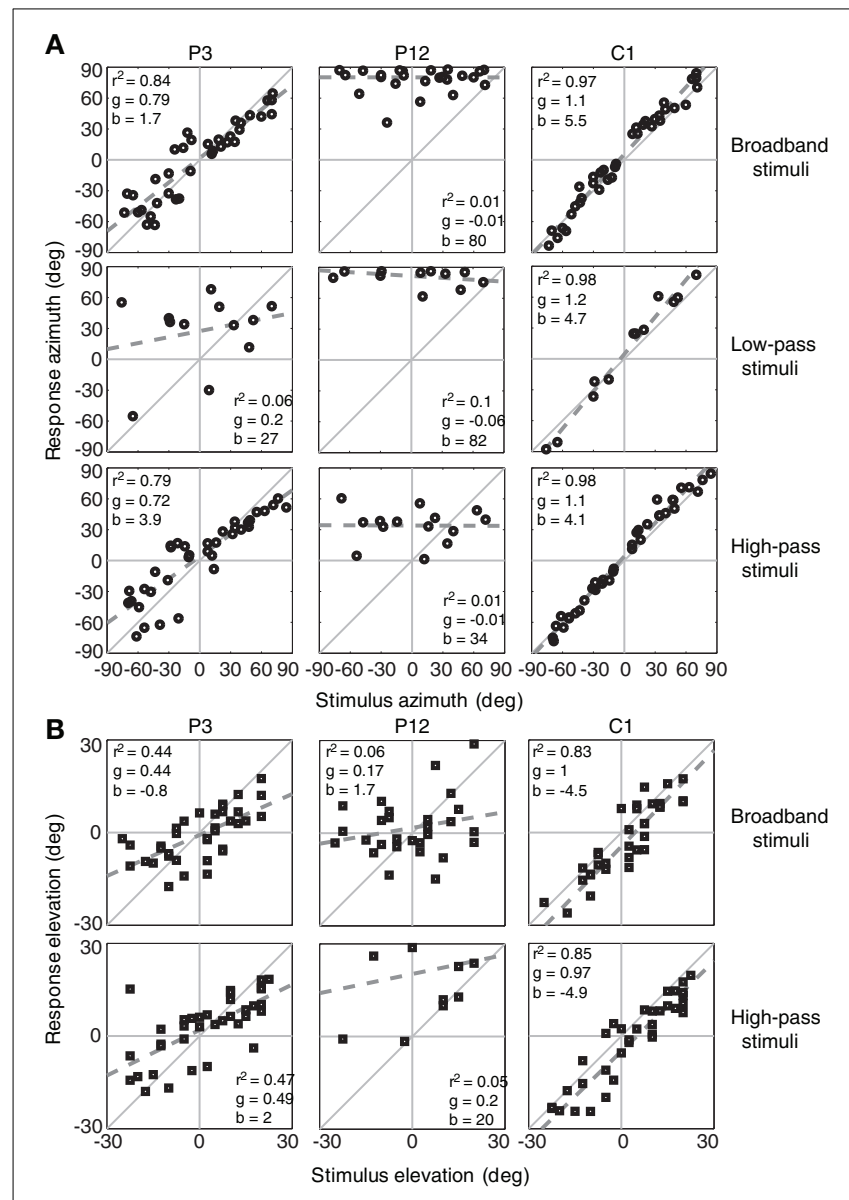

FIGURE 1 | Sound-localization responses for SSD listener with a thresholds at $\mathbf{8} \mathbf{~ k H z}<\mathbf{4 0 ~ d B ~ H L ~ ( P 3 ) , ~ a ~ S S D ~ l i s t e n e r ~ w i t h ~ a ~ t h r e s h o l d s ~}$ at $\mathbf{8 ~ k H z} \geq \mathbf{4 0 ~ d B ~} \mathbf{H L}$ (P12) and a control listener (C1). Responses are plotted for the BB, HP, and LP stimuli in azimuth (A) and elevation (B). The dashed gray line denotes the linear regression fit. Note the high degree of variation in monaural localization abilities of the listeners with SSD. Listener P3 had fairly good localization of BB and HP stimuli. $r^{2}$, coefficient of determination, $g$, response gain, $b$, bias.

at $8 \mathrm{kHz}$ in the better ear are listed in Table 1. Listener P3 demonstrated a $8 \mathrm{kHz}$ hearing threshold of $5 \mathrm{~dB}$ HL, P12 demonstrated a $8 \mathrm{kHz}$ threshold of $70 \mathrm{~dB}$ HL. Because of the high-frequency hearing loss listener P12 did not detect all stimuli.

Figure 1B shows the stimulus-response relations in elevation (Equation 4) for the same listeners. Listener P3, with better horizontal sound localization abilities than listener P12, demonstrated also better elevation performance $(g>0.44$ vs. $g<0.2)$.

Figure 2 shows the pooled azimuth stimulus-response relations of all control listeners $(n=15)$, all SSD listeners with $8 \mathrm{kHz}$ thresholds below $40 \mathrm{~dB} \mathrm{HL}(n=13)$, and SSD listeners with $8 \mathrm{kHz}$ thresholds higher than $40 \mathrm{~dB}$ HL $(n=6)$, for BB, LP and HP stimuli. If the right ear was the deaf ear, data are presented without modification. If the left ear was the deaf ear, data of left and right ears were swapped before pooling the data. The figure demonstrates that listeners without high-frequency hearing loss outperformed listeners with $8 \mathrm{kHz}$ thresholds higher than $40 \mathrm{~dB}$ HL, for BB and HP sounds. The figure hints at the possibility

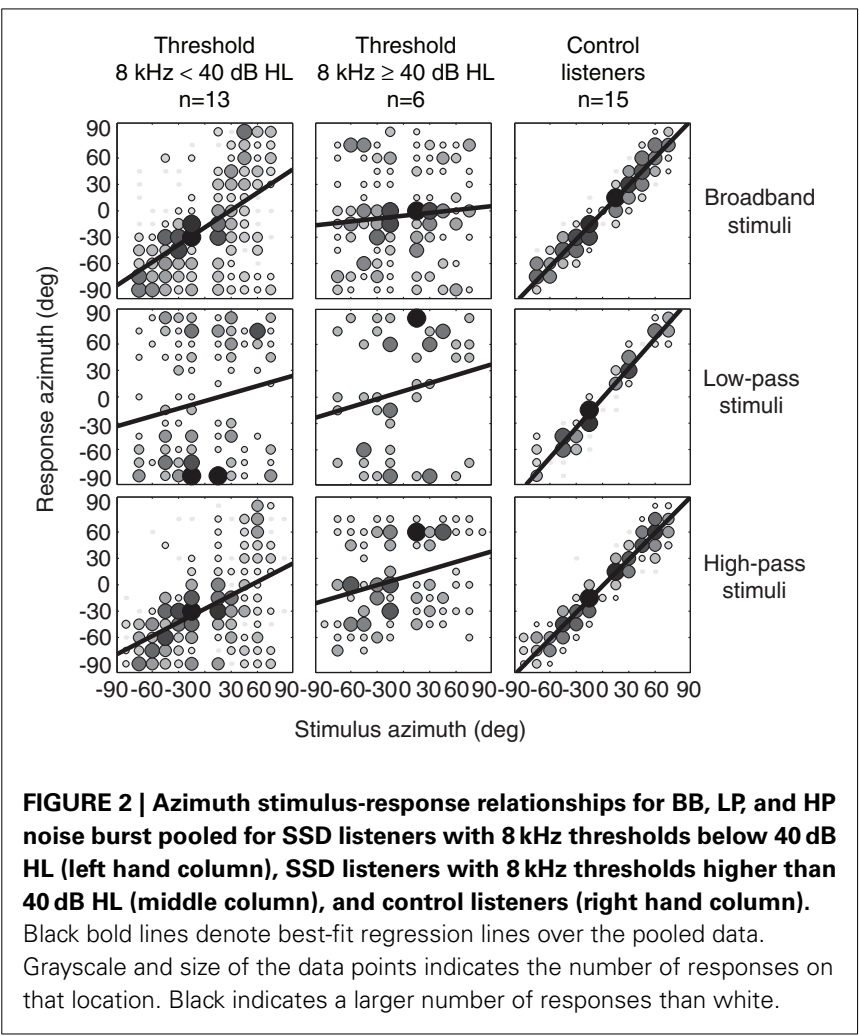

that SSD listeners with $8 \mathrm{kHz}$ thresholds below $40 \mathrm{~dB}$ HL were able to use spectral pinna-cues, as they could localize the $\mathrm{BB}$ and HP stimuli in azimuth, but were not able to localize the LP sounds. Listeners with $8 \mathrm{kHz}$ thresholds higher than $40 \mathrm{~dB} \mathrm{HL}$ were equally poor in localization of BB, LP, and HP stimuli.

Figure 3 shows the pooled stimulus-response relations in elevation. Listeners with $8 \mathrm{kHz}$ thresholds below $40 \mathrm{~dB} \mathrm{HL}$ outperformed listeners with $8 \mathrm{kHz}$ thresholds higher than $40 \mathrm{~dB} \mathrm{HL}$. The figure shows that SSD listeners with $8 \mathrm{kHz}$ thresholds below $40 \mathrm{~dB}$ HL were able to use spectral pinna-cues for the localization of $\mathrm{BB}$ and $\mathrm{HP}$ stimuli in elevation. Listeners with $8 \mathrm{kHz}$ thresholds higher than $40 \mathrm{~dB}$ HL were equally poor in localization of $\mathrm{BB}$ and HP stimuli. Two control listeners with high-frequency hearing loss (threshold $8 \mathrm{kHz}>40 \mathrm{~dB} \mathrm{HL}$ ) were not included in the pooled elevation stimulus-response relations (right hand column).

\section{CONTRIBUTION OF SPECTRAL CUES}

Figure 4 plots response azimuth localization gains for BB stimuli against response elevation gains for $13 \mathrm{SSD}$ listeners with $8 \mathrm{kHz}$ thresholds in the hearing ear below $40 \mathrm{~dB} \mathrm{HL}$ (filled symbols), six SSD listeners with $8 \mathrm{kHz}$ thresholds above $40 \mathrm{~dB} \mathrm{HL}$ (open circles), and the 15 control listeners (crosses).

Figure 4A shows the gains for the listeners with SSD in the monaural condition, and for the normal hearing control listeners (spectral-shape cues are available). Listeners with SSD demonstrated considerable variability in performance, and there was a clear correlation between azimuth gains and elevation gains ( $r=$ $0.83, p<0.01)$. The SSD listeners with $8 \mathrm{kHz}$ thresholds below 
$40 \mathrm{~dB}$ HL demonstrated higher azimuth and elevation gains than the SSD listeners with thresholds above $40 \mathrm{~dB}$ HL. The latter group of listeners had both gains close to zero, indicating poor directional hearing performance in both azimuth and elevation. The $r^{2}$ were also small ( $<0.4$, data not shown). The far majority of control listeners had azimuth and elevation gains that were close to the ideal value of one. The two older control listeners with high-frequency hearing loss demonstrated small elevation gains, confirming earlier reports of deteriorated vertical sound localization performance in the elderly (Otte et al., 2013).

Figure 4B shows the resulting azimuth and elevation gains when the molds reduced the spectral-shape cues $(r=0.2$, $p=0.87)$. Note that the SSD listeners with a relatively low

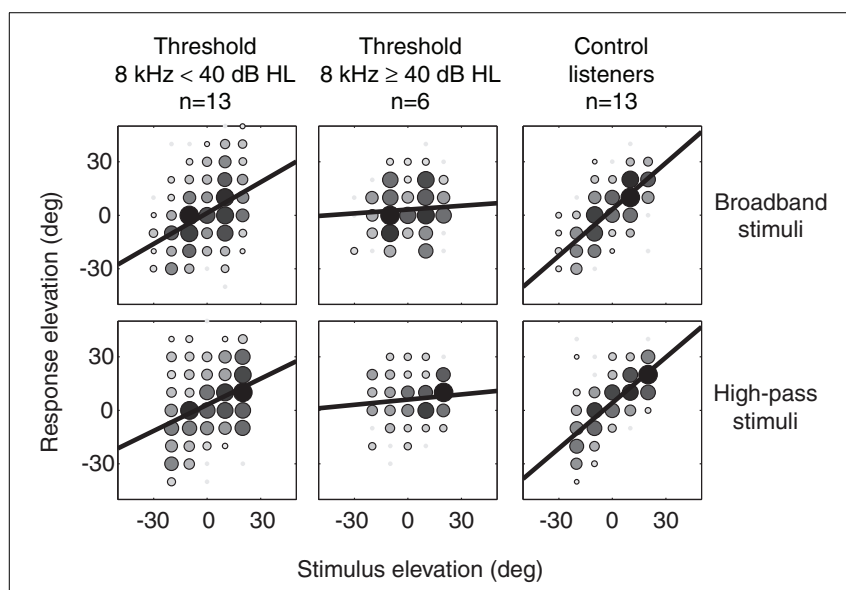

FIGURE 3 | Elevation stimulus-response relationships for BB and HP noise burst pooled for SSD listeners with $8 \mathrm{kHz}$ thresholds below $40 \mathrm{~dB}$ HL (left hand column), SSD listeners with $8 \mathrm{kHz}$ thresholds higher than $40 \mathrm{~dB}$ HL (middle column) and control listeners ( $n=13$, right hand column). Black bold lines denote best-fit regression lines over the pooled data. Grayscale and size of the data points indicates the number of responses on that location. Black indicates a larger number of responses than white. high-frequency hearing loss demonstrated a clear deterioration in their sound localization performance in both directions. Molds in the pinnae of control listeners only affected their elevation performance. This deterioration of sound localization abilities in elevation, after altering the pinna-cues with custom-made molds in both pinna, has been reported previously (Oldfield and Parker, 1984).

\section{CONTRIBUTION OF HIGH-FREQUENCY HEARING LOSS}

Figure 5 illustrates the effect of high-frequency $(8 \mathrm{kHz})$ hearing loss on the localization performance, of BB noises, of SSD listeners in the horizontal plane. When the hearing loss at $8 \mathrm{kHz}$ exceeds about $30 \mathrm{~dB}$ HL the azimuth gains are always small $(g<0.4)$. Good high-frequency hearing in the only hearing ear appears to be an important requirement for adequate sound localization performance. The variation in localization performance is not explained by the onset of unilateral deafness (congenital vs. acquired). In addition we also included the data of 9 listeners with SSD from the study of Van Wanrooij and Van Opstal (2004; squares). This figure clearly shows that almost half of the subjects with $8 \mathrm{kHz}$ thresholds below $40 \mathrm{~dB} \mathrm{HL}$ demonstrate poor sound localization abilities.

Elevation gains also clearly deteriorate with increasing highfrequency hearing loss. For all subjects with $8 \mathrm{kHz}$ thresholds above $40 \mathrm{~dB}$ HL elevation gains were small.

\section{EFFECT OF SOUND LEVEL ON LOCALIZATION PERFORMANCE}

Figure 6 shows the partial correlation coefficients for azimuth ( $p$ in Equation 2) and for the proximal sound level ( $q$ in Equation 2) for the BB stimuli, for SSD listeners (circles) and control listeners (crosses). These partial correlation coefficients reveal the relative contributions of the actual target azimuth and the perceived sound level at the hearing ear to their azimuth localization responses. For SSD listeners with an $8 \mathrm{kHz}$ threshold below $40 \mathrm{~dB}$, the contribution of proximal sound level varied systematically with the azimuth coefficient. Responses were more influenced by sound level when the (spectrally derived) estimate of azimuth

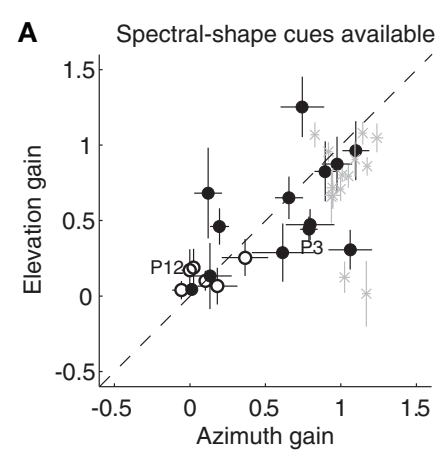

FIGURE 4 | Response elevation gain for BB stimuli plotted against the azimuth gain. Data from all control listeners (gray crosses), listeners with SSD with $8 \mathrm{kHz}$ thresholds below $40 \mathrm{~dB} H \mathrm{H}$ (filled circles) and SSD listeners with $8 \mathrm{kHz}$ thresholds higher than $40 \mathrm{~dB} \mathrm{HL}$ (open circles) are presented when spectral-shape cues were available (A), and when spectral-shape cues were reduced by molds (B). Error bars

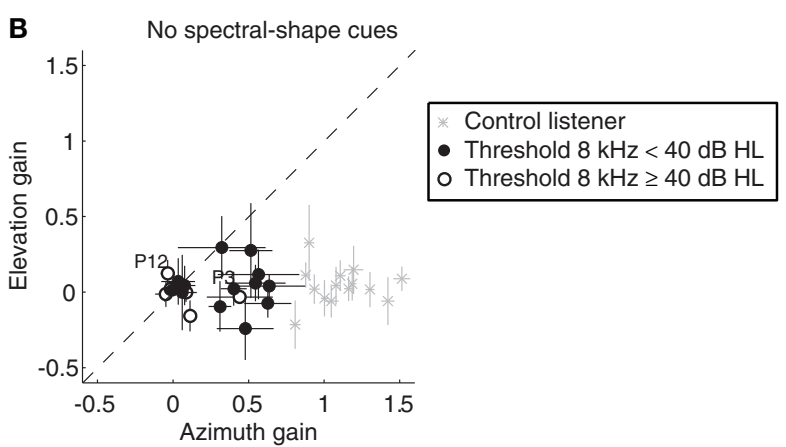

denote \pm 1 SE of the azimuth and elevation regression coefficients. Data points from the two SSD listeners depicted in Figure 1 (P3 and $\mathrm{P} 12)$, are indicated in the figure. Data are pooled across presentation levels. Note the two clear outliers in the control group. These two listeners demonstrated bilateral high-frequency hearing loss $(8 \mathrm{kHz}$ thresholds higher than $40 \mathrm{~dB} \mathrm{HL}$ ). 


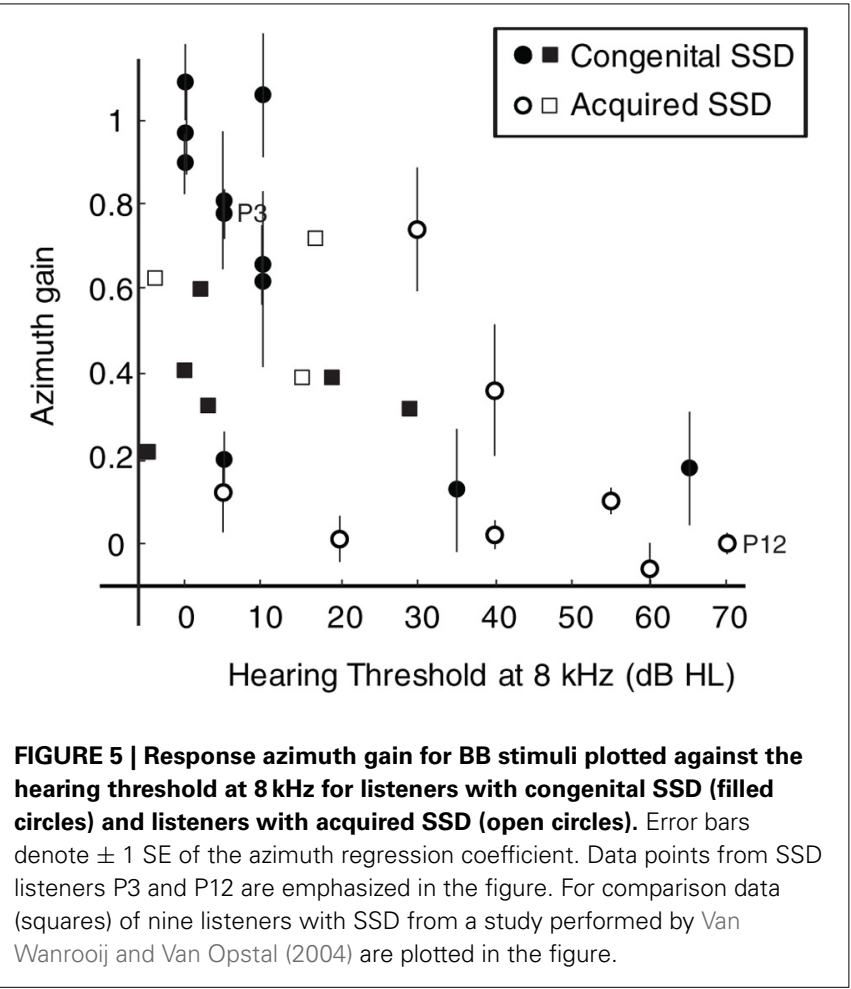

was poor. Indeed, those SSD listeners typically perceived louder sounds on their hearing side. A similar effect of sound level on localization performance in cochlear-implant listeners has been reported by Majdak et al. (2011).

Listener P12 is the listener with the most severe high-frequency hearing loss (see Table 1). This listener did not detect all BB stimuli and therefore proximal sound level did not contribute to the localization performance.

Control listeners had their azimuth coefficients close to the ideal value of one, and the proximal sound level coefficient close to zero. When listeners can localize sounds on the basis of binaural difference cues they rely less on the HSE cue.

\section{DISCUSSION}

\section{INDIVIDUAL DIFFERENCES IN SOUND LOCALIZATION PERFORMANCE}

The present study demonstrates that SSD listeners without severe high-frequency hearing loss in their hearing ear can localize BB noises in the horizontal plane. Our data indicate that the amount of high-frequency hearing loss greatly influences the directional hearing abilities of SSD listeners (Figure 5). Colburn (1982) postulated that the etiology of subjects with unilateral total deafness (e.g., congenital vs. acquired), may be irrelevant for their localization abilities. In support of this idea, our data indicate that the variability in localization performance of SSD listeners can to a large extent be attributed to high-frequency hearing loss, and not to the onset of unilateral deafness (congenital vs. acquired). However, good high-frequency hearing $(8 \mathrm{kHz}$ thresholds $<40 \mathrm{~dB}$ HL) does not always ensure good sound localization abilities. Even in the group of SSD listeners with $8 \mathrm{kHz}$ thresholds below $40 \mathrm{~dB} \mathrm{HL}$ almost half of the subjects demonstrate poor sound localization. This variation in sound localization performance

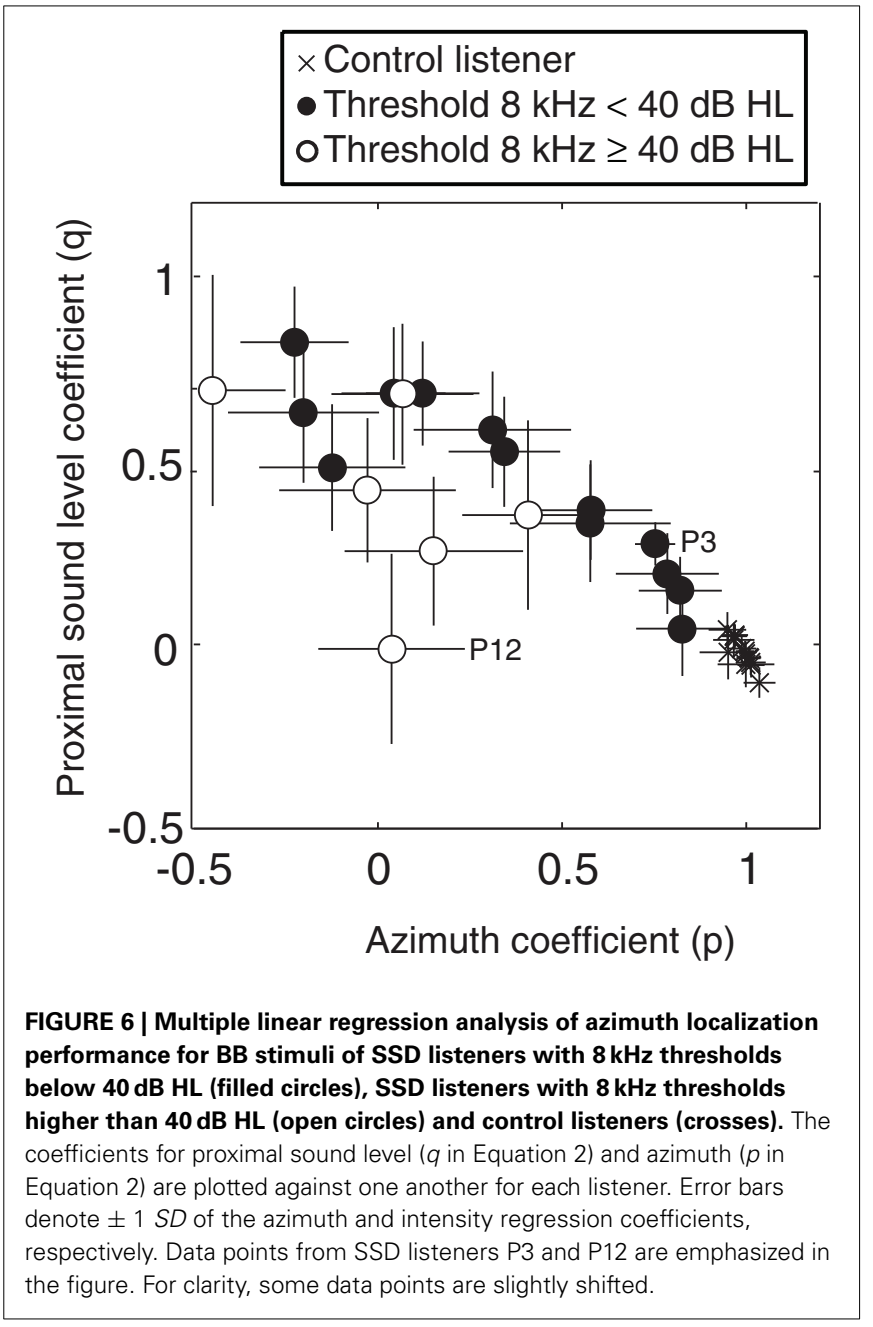

can be related to several factors. Recently, Andéol et al. (2013) and Majdak et al. (2014) demonstrated that in listeners with normal hearing, non-acoustic factors like the perceptual ability to discriminate spectral shapes had a larger impact on the sound localization performance in elevation than cues provided by the listener-specific pinna-induced spectral-shape cues. These non-acoustic factors might also play a role in the azimuthal localization abilities of SSD listeners.

\section{PINNA-INDUCED SPECTRAL-SHAPE CUES}

Some listeners with SSD were able to use the spectral-pinna cues of their hearing ear for localization in azimuth. When the possibility to use spectral cues was disrupted by filling the pinna of their hearing ear with a mold, azimuthal localization deteriorated (Figure 4B). The spectral cues are specific for an individual listener and appear above about $4 \mathrm{kHz}$ (Batteau, 1967; Middlebrooks and Green, 1991). BB noises can be localized in the vertical plane, because the brain can dissociate the elevation dependent pinna-induced spectral shape cues. Apparently, azimuth dependent changes in the spectral cues are used when the auditory system is deprived from binaural cues. Recently Otte et al. (2013) demonstrated that the pinna-induced 
spectral shape cues are changing during life because the ears keep growing, and that listeners adapt to this changing cues. A limitation of the present study is that we did not measure the spectral cues in terms of head-related transfer functions (HRTFs) or non-acoustic factors like the perceptual ability to discriminate spectral shapes (Andéol et al., 2013; Majdak et al., 2014) of the SSD listeners.

\section{INCREASING NUMBER OF TREATMENT OPTIONS FOR SSD}

Studies have shown that children with SSD demonstrate worse language scores compared to their normal-hearing peers, and that they are at risk for learning problems in school (Lieu, 2013). There is increasing evidence that adults with SSD experience problems in social settings because of their disability in binaural processing (Wie et al., 2010).

The criteria for treatment of SSD are expanding, and more treatment options become available. One treatment option is to provide a contralateral routing of sound (CROS) device. These devices transmit sounds presented at the deaf side to the hearing ear. Currently, the two most commonly applied CROS interventions are the wireless conventional CROS hearing aid, and the percutaneous bone-conduction hearing device (Bosman et al., 2003). Although listeners with SSD have only a single functioning cochlea, and therefore bone-conduction would not restore binaural hearing, the bone-conduction device is offered more often as an option for rehabilitation (Spitzer et al., 2002; Hol et al., 2004; Newman et al., 2008; Grantham et al., 2012; Nicolas et al., 2012; Battista et al., 2013).

In several countries cochlear implantation has become a treatment option (Arndt et al., 2011; Kamal et al., 2012; Arnoldner and Lin, 2013), and it is even proposed to implant children with congenital SSD already at a young age (Tzifa and Hanvey, 2013). Potentially, this option can lead to binaural hearing.

\section{CONCLUSION}

The present study emphasizes the importance of a precise evaluation of the monaural hearing abilities of listeners with SSD, especially at the higher frequencies for which the spectral-shape cues become unambiguous for sound localization. Some SSD listeners were using monaural pinna-induced spectral-shape cues of their hearing ear, for localization of BB noises in both azimuth and elevation. Because spectral cues are minimal for LP noises (Middlebrooks, 1992; Blauert, 1997) these stimuli could not be localized by SSD listeners. For clinicians it might be important to understand the factors affecting the localization performance of SSD listeners in order to give the hearing impaired the best advice in case of desired treatment.

\section{ACKNOWLEDGMENTS}

This research was funded by the William Demants og Hustru Ida Emilies Fond (Martijn J. H. Agterberg), the Radboud University Nijmegen (A. John Van Opstal), the Donders Institute for Brain, Cognition and Behaviour (Martijn J. H. Agterberg, Marc M. Van Wanrooij), and the Department of Otorhinolaryngology at the Radboud University Medical Centre Nijmegen (Ad F. M. Snik, Myrthe K. S. Hol). We thank Chris-Jan Beerendonk and Gunter Windau for their technical support.

\section{REFERENCES}

Agterberg, M. J., Snik, A. F., Hol, M. K., Van Wanrooij, M. M., and Van Opstal, A. J. (2012). Contribution of monaural and binaural cues to sound localization in patients with unilateral conductive hearing loss; improved directional hearing with a bone-conduction device. Hear. Res. 286, 9-18. doi: 10.1016/j.heares.2012.02.012

Andéol, G., Macpherson, E. A., and Sabin, A. T. (2013). Sound localization in noise and sensitivity to spectral shape. Hear. Res. 304, 20-27. doi: 10.1016/j.heares.2013.06.001

Arndt, S., Aschendorff, A., Laszig, R., Beck, R., Schild, C., Kroeger, S., et al. (2011). Comparison of pseudobinaural hearing to real binaural hearing rehabilitation after cochlear implantation in patients with unilateral deafness and tinnitus. Otol. Neurotol. 32, 39-47. doi: 10.1097/MAO.0b013e3181fcf271

Arnoldner, C., and Lin, V. Y. (2013). Expanded selection criteria in adult cochlear implantation. Cochlear Implants Int. 14(Suppl. 4), 10-13. doi: 10.1179/1467010013Z.000000000123

Batteau, D. W. (1967). The role of the pinna in human localization. Proc. R. Soc. Lond. B Biol. Sci. 168, 158-180. doi: 10.1098/rspb.1967.0058

Battista, R. A., Mullins, K., Wiet, R. M., Sabin, A., Kim, J., and Rauch, V. (2013). Sound localization in unilateral deafness with the Baha or TransEar device. JAMA Otolaryngol. Head Neck Surg. 139, 64-70. doi: 10.1001/jamaoto.2013.1101

Best, V., Carlile, S., Jin, C., and van Schaik, A. (2005). The role of high frequencies in speech localization. J. Acoust. Soc. Am. 118, 353-363. doi: 10.1121/1.1926107

Blauert, J. (1997). Spatial Hearing. The Psychophysics of Human Sound Localization. Cambridge, MA: MIT.

Bosman, A. J., Hol, M. K., Snik, A. F., Mylanus, E. A., and Cremers, C. W. (2003). Bone-anchored hearing aids in unilateral inner ear deafness. Acta Otolaryngol. 123, 258-260. doi: 10.1080/000164580310001105

Bremen, P., Van Wanrooij, M. M., and Van Opstal A. J. (2010). Pinna cues determine orienting response modes to synchronous sounds in elevation. J. Neurosci. 30, 194-204. doi: 10.1523/JNEUROSCI.2982-09.2010

Colburn, H. S. (1982). Binaural interaction and localization with various hearing impairments. Scand. Audiol. Suppl. 15, 27-45.

Frens, M. A., and Van Opstal, A. J. (1995). A quantitative study of auditory-evoked saccadic eye movements in two dimensions. Exp. Brain Res. 107, 103-117. doi: 10.1007/BF00228022

Grantham, D. W., Ashmead, D. H., Haynes, D. S., Hornsby, B. W., Labadie, R. F., and Ricketts, T. A. (2012). Horizontal plane localization in single-sided deaf adults fitted with a bone-anchored hearing aid (Baha). Ear Hear. 33, 595-603. doi: 10.1097/AUD.0b013e3182503e5e

Häusler, R., Colburn, S., and Marr, E. (1983). Sound localization in subjects with impaired hearing. Spatial-discrimination and interaural-discrimination tests. Acta Otolaryngol. Suppl. 400, 1-62. doi: 10.3109/00016488309105590

Hofman, P. M., and Van Opstal, A. J. (1998). Spectro-temporal factors in twodimensional human sound localization. J. Acoust. Soc. Am. 103, 2634-2648. doi: $10.1121 / 1.422784$

Hol, M. K., Bosman, A. J., Snik, A. F., Mylanus, E. A., and Cremers, C. W. (2004). Bone-anchored hearing aid in unilateral inner ear deafness: a study of 20 patients. Audiol. Neurootol. 9, 274-281. doi: 10.1159/000080227

Humes, L. E., Allen, S. K., and Bess, F. H. (1980). Horizontal sound localization skills of unilaterally hearing-impaired children. Audiology 19, 508-518. doi: $10.3109 / 00206098009070082$

Irving, S., and Moore, D. R. (2011). Training sound localization in normal hearing listeners with and without a unilateral ear plug. Hear. Res. 280, 100-108. doi: 10.1016/j.heares.2011.04.020

Kamal, S. M., Robinson, A. D., and Diaz, R. C. (2012). Cochlear implantation in single-sided deafness for enhancement of sound localization and speech perception. Curr. Opin. Otolaryngol. Head Neck Surg. 20, 393-397. doi: 10.1097/MOO.0b013e328357a613

Keating, P., Dahmen, J. C., and King, A. J. (2013). Context-specific reweighting of auditory spatial cues following altered experience during development. Curr. Biol. 23, 1291-1299. doi: 10.1016/j.cub.2013.05.045

Kral, A., Hubka, P., Heid, S., and Tillein, J. (2013). Single-sided deafness leads to unilateral aural preference within an early sensitive period. Brain 136, 180-193. doi: 10.1093/brain/aws305

Kumpik, D. P., Kacelnik, O., and King, A. J. (2010). Adaptive reweighting of auditory localization cues in response to chronic unilateral earplugging in humans. J. Neurosci. 30, 4883-4894. doi: 10.1523/JNEUROSCI.5488-09.2010 
Lieu, J. E. (2013). Unilateral hearing loss in children: speech-language and school performance. B-ENT 21, 107-115.

Majdak, P., Baumgartner, R., and Laback, B. (2014). Acoustic and non-acoustic factors in modeling listener-specific performance of sagittal-plane sound localization. Front. Psychol. 5:319. doi: 10.3389/fpsyg.2014.00319

Majdak, P., Goupell, M. J., and Laback, B. (2011). Two-dimensional localization of virtual sound sources in cochlear-implant listeners. Ear Hear. 32, 198-208. doi: 10.1097/AUD.0b013e3181f4dfe9

McPartland, J. L., Culling, J. F., and Moore, D. R. (1997). Changes in lateralization and loudness judgements during one week of unilateral ear plugging. Hear. Res. 113, 165-172. doi: 10.1016/S0378-5955(97)00142-1

Middlebrooks, J. C. (1992). Narrow-band sound localization related to external ear acoustics. J. Acoust. Soc. Am. 92, 2607-2624. doi: 10.1121/1.404400

Middlebrooks, J. C., and Green, D. M. (1991). Sound localization by human listeners. Annu. Rev. Psychol. 42, 135-159. doi: 10.1146/annurev.ps.42.020191. 001031

Newman, C. W., Sandridge, S. A., and Wodzisz, L. M. (2008). Longitudinal benefit from and satisfaction with the Baha system for patients with acquired unilateral sensorineural hearing loss. Otol. Neurotol. 29, 1123-1131. doi: 10.1097/MAO.0b013e31817dad20

Nicolas, S., Mohamed, A., Yoann, P., Laurent, G., and Thierry, M. (2012). Longterm benefit and sound localization in patients with single-sided deafness rehabilitated with an osseointegrated bone-conduction device. Otol. Neurotol. 34, 111-114. doi: 10.1097/MAO.0b013e31827a2020

Oldfield, S. R., and Parker, S. P. (1984). Acuity of sound localisation: a topography of auditory space. II. Pinna cues absent. Perception 13, 601-617. doi: 10.1068/p130601

Otte, R. J., Agterberg, M. J., Van Wanrooij, M. M., Snik, A. F., and Van Opstal, A.J. (2013). Age-related hearing loss and ear morphology affect vertical but not horizontal sound-localization performance. J. Assoc. Res. Otolaryngol. 14, 261-273. doi: 10.1007/s10162-012-0367-7

Robinson, D. A. (1963). A method of measuring eye movements using a sclera search coil in a magnetic field. IEEE Trans. Biomed. Eng. 10, 137-145.

Rothpletz, A. M., Wightman, F. L., and Kistler, D. J. (2012). Informational masking and spatial hearing in listeners with and without unilateral hearing loss. J. Speech Lang. Hear. Res. 55, 511-531. doi: 10.1044/1092-4388(2011/10-0205)

Shub, D. E., Carr, S. P., Kong, Y., and Colburn, H. S. (2008). Discrimination and identification of azimuth using spectral shape. J. Acoust. Soc. Am. 124, 3132-3141. doi: 10.1121/1.2981634

Slattery, W. H. III., and Middlebrooks, J. C. (1994). Monaural sound localization: acute versus chronic unilateral impairment. Hear. Res. 75, 38-46. doi 10.1016/0378-5955(94)90053-1
Spitzer, J. B., Ghossaini, S. N., and Wazen, J. J. (2002). Evolving applications in the use of bone-anchored hearing aids. Am. J. Audiol. 11, 96-103. doi: $10.1044 / 1059-0889(2002 / 011)$

Tzifa, K., and Hanvey, K. (2013). Cochlear implantation in asymmetrical hearing loss for children: our experience. Cochlear Implants Int. 14(Suppl. 4), 56-61. doi: 10.1179/1467010013Z.000000000137

Van Wanrooij, M. M., and Van Opstal, A. J. (2004). Contribution of head shadow and pinna cues to chronic monaural sound localization. J. Neurosci. 24, 4163-4171. doi: 10.1523/JNEUROSCI.0048-04.2004

Van Wanrooij, M. M., and Van Opstal, A. J. (2007). Sound localization under perturbed binaural hearing. J. Neurophys. 97, 715-726. doi: 10.1152/jn.00260.2006

Van Wieringen, A., De Voecht, K., Bosman, A. J., and Wouters, J. (2011). Functional benefit of the bone-anchored hearing aid with different auditory profiles: objective and subjective measures. Clin. Otolaryngol. 36, 114-120. doi: 10.1111/j.1749-4486.2011.02302.x

Wazen, J. J., Ghossaini, S. N., Spitzer, J. B., and Kuller, M. (2005). Localization by unilateral BAHA users. Otolaryngol. Head Neck Surg. 132, 928-932. doi: 10.1016/j.otohns.2005.03.014

Wie, O. B., Pripp, A. H., and Tvete, O. (2010). Unilateral deafness in adults: effects on communication and social interaction. Ann. Otol. Rhinol. Laryngol. 119, 772-781.

Wightman, F. L., and Kistler, D. J. (1997). Monaural sound localization revisited. J. Acoust. Soc. Am. 101, 1050-1063. doi: 10.1121/1.418029

Conflict of Interest Statement: The authors declare that the research was conducted in the absence of any commercial or financial relationships that could be construed as a potential conflict of interest.

Received: 13 April 2014; paper pending published: 05 May 2014; accepted: 13 June 2014; published online: 04 July 2014.

Citation: Agterberg MJH, Hol MKS, Van Wanrooij MM, Van Opstal AJ and Snik AFM (2014) Single-sided deafness and directional hearing: contribution of spectral cues and high-frequency hearing loss in the hearing ear. Front. Neurosci. 8:188. doi: 10.3389/ fnins.2014.00188

This article was submitted to Auditory Cognitive Neuroscience, a section of the journal Frontiers in Neuroscience.

Copyright (๑) 2014 Agterberg, Hol, Van Wanrooij, Van Opstal and Snik. This is an open-access article distributed under the terms of the Creative Commons Attribution License (CC BY). The use, distribution or reproduction in other forums is permitted, provided the original author(s) or licensor are credited and that the original publication in this journal is cited, in accordance with accepted academic practice. No use, distribution or reproduction is permitted which does not comply with these terms. 